TITLE:

\title{
A Bayesian approach for vibration- based long-term bridge monitoring to consider environmental and operational changes
}

\section{AUTHOR(S):}

Kim, Chul-Woo; Morita, Tomoaki; Oshima, Yoshinobu; Sugiura, Kunitomo

\section{CITATION:}

Kim, Chul-Woo ... [et al]. A Bayesian approach for vibration-based long-term bridge monitoring to consider environmental and operational changes. Smart Structures and Systems 2015, 15(2): 395-408

ISSUE DATE:

2015-02-25

URL:

http://hdl.handle.net/2433/236010

RIGHT:

発行元の許可を得て掲載しています。This is not the published version. Please cite only the published version. この論文は出版社版でありませ ん。引用の際には出版社版をご確認ご利用ください。 


\title{
A Bayesian approach for vibration-based long-term bridge monitoring to consider environmental and operational changes
}

\author{
Chul-Woo Kim¹, Tomoaki Morita ${ }^{1 \mathrm{a}}$, Yoshinobu Oshima ${ }^{1 \mathrm{~b}}$ \\ and Kunitomo Sugiura ${ }^{1 \mathrm{c}}$ \\ ${ }^{1}$ Department of Civil and Earth Resources Engineering, Graduate School of Engineering, \\ Kyoto University, Kyotodaigaku Katsura, Nishikyo-ku, Kyoto 615-8540, Japan
}

(Received

, Revised

, Accepted

)

\begin{abstract}
This study aims to propose a Bayesian approach to consider changes in temperature and vehicle weight as environmental and operational factors for vibration-based long-term bridge health monitoring. The Bayesian approach consists of three steps: step 1 is to identify damage-sensitive features from coefficients of the autoregressive model utilizing bridge accelerations; step 2 is to perform a regression analysis of the damage-sensitive features to consider environmental and operational changes by means of the Bayesian regression; and step 3 is to make a decision on the bridge health condition based on residuals, differences between the observed and predicted damage-sensitive features, utilizing 95\% confidence interval and the Bayesian hypothesis testing. Feasibility of the proposed approach is examined utilizing monitoring data on an in-service bridge recorded over a one-year period. Observations through the study demonstrated that the Bayesian regression considering environmental and operational changes led to more accurate results than that without considering environmental and operational changes. The Bayesian hypothesis testing utilizing data from the healthy bridge, the damage probability of the bridge was judged as no damage.
\end{abstract}

Keywords: long-term bridge monitoring; Bayesian regression; temperature; vehicle weight; vibration

\section{Introduction}

Maintaining and improving civil infrastructures including bridge structures are keen technical issues in many countries. Developing an effective maintenance strategy relies on a timely decision on the health condition of the structure. Structural health monitoring (SHM) using vibration data thus has been recognized as one of the promising technologies for providing a timely decision on the bridge health condition. Most precedent studies on SHM specifically examine changes in modal properties of structures (e.g. Doebling et al. 1996). The fundamental concept of this technology is that modal parameters are functions of structures' physical properties. Therefore, a

\footnotetext{
${ }^{*}$ Corresponding author, Professor, E-mail: kim.chulwoo.5u@kyoto-u.ac.jp

${ }^{a}$ Master Student, E-mail: morita.tomoaki.56c@st.kyoto-u.ac.jp

b Associate Professor, E-mail: oshima.yoshinobu.3a@kyoto-u.ac.jp

c Professor, E-mail:sugiura.kunitomo.4n@kyoto-u.ac.jp
} 
change in physical properties, such as reduced stiffness resulting from damage, will detectably change these modal properties. In fact, many techniques to identify the hidden information of structural integrity in the vibration data have been proposed to diagnose bridge structures (Deraemaeker et al. 2007, Dilena and Morassi 2011 and Kim et al. 2012). However, real bridge structures are subject to changing environmental and operational conditions that affect structural integrity information during the long in-service period. Some components in the signals affected by those environmental and operational effects lurk in the measured vibration data and disguise themselves as structural responses (Sohn et al. 2003, and Deraemaeker et al. 2007). As in-service effects on vibration monitoring of bridges, temperature, wind-induced and traffic-induced vibrations and traffic mass effects are factors to be considered. Focusing on short and medium span bridges, however, temperature and traffics are dominant factors affecting the vibration of bridges (Peeters and De Roeck 2001, and Cunha et al. 2013). Therefore, how to consider those environmental and operational effects in long-term bridge health monitoring is a crucial issue.

This study is intended to investigate a way to consider time-varying temperature and vehicle weight as environmental and operational factors for long-term vibration-based bridge health monitoring (BHM) by means of a Bayesian approach, which is an improvement from previous researches considering only temperature as an environmental factor by Kim et al. (2011 and 2013a).

The proposed Bayesian approach consists of three steps. Step 1 is to identify damage-sensitive features (damage indicators, DIs) from coefficients of the auto-regressive (AR) model utilizing bridge accelerations (Nair et al. 2006, Kim et al. 2012 and 2013b). Since AR coefficients are closely linked with features of bridge vibrations, the DI changes due to changes in the bridge health condition as well as environmental and operational conditions during the monitoring campaign. Step 2 is to perform a regression analysis of the DIs identified in step 1 (observed DIs (DI ${ }_{\mathrm{ob}}$ 's)) to consider environmental and operational changes by means of the Bayesian regression (Kitagawa and Gersch 1984). The Bayesian regression is useful to examine long-term monitoring data effectively by online updating. Step 3 is to make a decision on the bridge health condition based on residuals utilizing 95\% confidence interval and the Bayesian hypothesis testing (Sankararaman and Mahadevan 2011). The residuals are differences between the DI ${ }_{o b}$ 's and the DI predicted by means of the Bayesian regression in step 2 (predicted DIs (DI pr's)).

The Bayesian approach is applied to investigate monitoring data of an in-service bridge. The data were measured at a seven-span plate-Gerber bridge recorded over a one-year period. This study considers time-varying temperature and vehicle weight as environmental and operational factors respectively. Vehicle weights are identified utilizing a bridge weigh-in-motion (BWIM) system (Moses 1979 and Heng et al. 2011) installed on the bridge. All the data is taken from the healthy bridge, since no damage and deterioration was reported during the monitoring period. The influence of time-varying environmental and operational factors is investigated by comparing three cases: Case 1 is to utilize acceleration, temperature and vehicle weight data considering temperature and vehicle weight as environmental and operational factors; Case 2 is to utilize acceleration and temperature data considering temperature as environmental factor; and Case 3 is to utilize only acceleration data without considering environmental and operational changes. 


\section{A Bayesian approach for long-term BHM to consider environmental changes}

\subsection{Identification of damage-sensitive features from AR coefficients: step 1}

Many studies focus on changes in system frequencies and structural damping constants for the structural diagnosis of bridges by utilizing a linear time series model such as the AR model (e.g. Kim et al. 2012, 2013c). However, there exist drawbacks in modal parameter-based bridge diagnosis using time series models; e.g., the optimal time series model for vibration responses of bridge structures usually comprises a higher-order term, and as a result the optimal model identifies even spurious modal parameters, which causes false system frequencies and damping constants. Those false modal parameters make it difficult to choose the proper modal parameters affected by structural damage. The drawback of the classical method is the driving force behind this study. This study considered an alternative parameter based on AR coefficients as a damagesensitive feature for the vibration-based BHM because both system frequency and damping constant are related to AR coefficients.

Step 1 is to identify the $\mathrm{DI}_{\mathrm{ob}}$ from coefficients of the AR model of bridge acceleration responses. This paper includes only a brief description about the DI without covering details, since theoretical backgrounds of utilizing AR coefficients as a damage-sensitive feature have already investigated by Nair et al. (2006) for a model building structure and by Kim et al. (2013b, 2013c) for a model bridge and thus details can be fully accessed in the studies.

Linear dynamic system can be idealized using the AR model shown in Eq. (1) (Kim et al. 2012, 2013c).

$$
z_{k}=\sum_{i=1}^{p} a_{i} z_{k-i}+e_{k}
$$

where $\mathrm{z}_{k}$ denotes the $k$-th output of a system, $a_{i}$ is the $i$-th AR coefficient, $p$ is the optimal AR order and $e_{k}$ indicates the $k$-th error. The optimal AR order, which is obtainable by means of Akaike Information Criteria (AIC) (Akaike 1974), is used in this study. AIC is given by Eq. (2)

$$
\mathrm{AIC}=n \log \left(2 \pi E^{2}\right)+2(m+1)+n
$$

where $n$ indicates the number of data, $m$ represents AR order, and $E^{2}$ means square of prediction error. The AIC consists of two terms; the first term is a log-likelihood function and the second term is a penalty function for the number of the AR order.

The $\mathrm{DI}_{\mathrm{ob}}$ is defined by Eq. (3) (Nair et al. 2006 and Kim et al. 2013c).

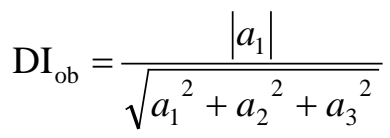

where $a_{1}, a_{2}$ and $a_{3}$ indicate the first, second and third AR coefficients respectively. Since the AR coefficients are closely linked with bridge vibrations, the $\mathrm{DI}_{\mathrm{ob}}$ changes due to changes in bridge health condition as well as environmental and operational conditions. Nair et al. (2006) show that the first three AR coefficients are the most significant among all the coefficients of the AR model utilizing data from the laboratory experiment on a model building. Kim et al. (2013b) also observes that the $\mathrm{DI}_{\mathrm{ob}}$, considering up to the third order of the AR coefficients, is a promising 
parameter in bridge health monitoring, since the $\mathrm{DI}_{\mathrm{ob}}$ is observed to be the most sensitive to damage through a bridge-moving vehicle laboratory experiment.

\subsection{Regression analysis to consider environmental changes by Bayesian regression: step 2}

\subsubsection{Introduction}

In a long-term bridge monitoring, the $\mathrm{DI}_{\mathrm{ob}}$ varies due to time-varying environmental and operational conditions. This study adopts the Bayesian regression to cope with influences of these time-varying environmental and operational conditions on the $\mathrm{DI}_{\mathrm{ob}}$ 's. The idea is to predict DIs $\left(\mathrm{DI} \mathrm{pr}_{\mathrm{r}}\right.$ considering environmental and operational effects in a regression model. If $\mathrm{DI}_{\mathrm{pr}}$ are comparable with $\mathrm{DI}_{\mathrm{ob}}$ 's then the residual that is the difference between the $\mathrm{DI}_{\mathrm{pr}}$ and $\mathrm{DI}_{\mathrm{ob}}$ will be small enough. In other words the residuals are fitted within a confidence interval statistically. Therefore this study monitors statistical changes of residuals of newly observed $\mathrm{DI}_{\mathrm{ob}}$ 's. If the residual of the $\mathrm{DI}_{\mathrm{ob}}$ 's crosses a confidence level, it can be treated as a potential anomaly.

Step 2 is to perform a regression analysis of the $\mathrm{DI}_{\mathrm{ob}}$ 's identified in the step 1 to consider influences from environmental and operational factors by means of the Bayesian regression as an online updating method (Kitagawa and Gersch 1984).

\subsubsection{Bayesian regression algorithm}

The state space model for the Bayesian regression is given by Eq. (4) and Eq. (5).

$$
\begin{aligned}
\mathbf{x}_{t} & =\mathbf{F} \mathbf{x}_{t-1}+\mathbf{G} \mathbf{v}_{t} \\
y_{t} & =\mathbf{H}_{t} \mathbf{x}_{t}+w_{t}
\end{aligned}
$$

where $\mathbf{x}_{t}$ and $y_{t}$ are the state matrix and observation at a time $t$ respectively. $\mathbf{v}_{t}$ and $w_{t}$ denote noise at $t$. For any particular model of the time series, $\mathbf{F}, \mathbf{G}$ and $\mathbf{H}_{t}$ are known. The future state can be predicted in terms of the Kalman filter as (Kitagawa and Gersch 1984)

$$
\begin{gathered}
\overline{\mathbf{x}}_{t \mid t-1}=\mathbf{F} \overline{\mathbf{x}}_{t-1 \mid t-1} \\
\mathbf{V}_{t \mid t-1}=\mathbf{F} \mathbf{V}_{t-1 \mid t-1} \mathbf{F}^{\mathrm{T}}+\mathbf{G Q G}^{\mathrm{T}}
\end{gathered}
$$

where $\overline{\mathbf{x}}_{t \mid t-1}$ and $\mathbf{V}_{t \mid t-1}$ denote the predicted conditional mean matrix and covariance matrix at $t$ under the condition of the state at $t-1$ respectively. $\mathbf{Q}$ stands for the covariance matrix of $\mathbf{v}_{t}$. The filtered state also can be estimated as

$$
\begin{gathered}
\overline{\mathbf{x}}_{t \mid t}=\overline{\mathbf{x}}_{t \mid t-1}+\mathbf{K}_{t}\left(y_{t}-\mathbf{H}_{t} \overline{\mathbf{x}}_{t \mid t-1}\right) \\
\mathbf{V}_{t \mid t}=\left(\mathbf{I}-\mathbf{K}_{t} \mathbf{H}_{t}\right) \mathbf{V}_{t \mid t-1}
\end{gathered}
$$

where $\mathbf{K}_{t}$ is the Kalman gain defined by Eq. (10).

$$
\mathbf{K}_{t}=\mathbf{V}_{t \mid t-1} \mathbf{H}_{t}^{\mathrm{T}}\left(\mathbf{H}_{t} \mathbf{V}_{t \mid t-1} \mathbf{H}_{t}^{\mathrm{T}}+R\right)^{-1}
$$

where $R$ denotes the variance of $w_{t}$. This study yields recursive computations for filtered estimates of the state matrix, and calculates predictions and residuals. Prediction at $t\left(\hat{y}_{t}\right)$ are defined by Eq. (11). 


$$
\hat{y}_{t}=\mathbf{H}_{t} \overline{\mathbf{x}}_{t \mid t}
$$

Residuals at $t\left(r_{t}\right)$, difference between the observation and prediction at $t$, are defined by Eq. (12).

$$
r_{t}=y_{t}-\hat{y}_{t}
$$

\subsubsection{Application to this study}

This study adopts $\mathrm{DI}_{\mathrm{ob}}$ at $t\left(\mathrm{DI}_{\mathrm{ob}}^{(t)}\right)$ as $y_{t}$ and $\mathrm{DI}_{\mathrm{pr}}$ at $t\left(\mathrm{DI}_{\mathrm{pr}}^{(t)}\right)$ as $\hat{y}_{t}$ respectively. Moreover, this study adopts a time-series model to consider environmental factors defined by Eq. (13).

$$
\mathrm{DI}_{\mathrm{ob}}^{(t)}=\sum_{i=1}^{q} \alpha_{i} \mathrm{DI}_{\mathrm{ob}}^{(t-i)}+\sum_{i=1}^{q} \beta_{i} u^{(t-i)}+w_{t}
$$

where $q$ stands for model order. $\alpha_{i}$ and $\beta_{i}$ are model parameters. $u^{(t)}$ and $w_{t}$ denote environmental and operational factors and noise at $t$ respectively. In other words, this study adopts the following relationships for the parameter and matrixes in Eq. (4) and Eq. (5).

$$
\begin{gathered}
y_{t}=\mathrm{DI}_{\mathrm{ob}}^{(t)} \\
\mathbf{F}=\mathbf{I} \quad \text { (Identity matrix) } \\
\mathbf{G}=\mathbf{0} \quad \text { (Null matrix) } \\
\mathbf{x}_{t}=\left[\alpha_{1}, \ldots, \alpha_{q}, \beta_{1}, \ldots, \beta_{q}\right] \\
\mathbf{H}_{t}=\left[\mathrm{DI}_{\mathrm{ob}}^{(t-1)}, \ldots, \mathrm{DI}_{\mathrm{ob}}^{(t-q)}, u^{(t-1)}, \ldots, u^{(t-q)}\right] \\
R=1
\end{gathered}
$$

Moreover the initial mean and covariance matrixes can be described as

$$
\begin{aligned}
& \overline{\mathbf{x}}_{0 \mid 0}=\mathbf{0} \text { (Null matrix) } \\
& \mathbf{V}_{0 \mid 0}=\mathbf{I} \text { (Identity matrix) }
\end{aligned}
$$

The flowchart of the Bayesian regression analysis to consider environmental changes in this study is shown in Fig. 1.

\subsection{Decision-making on bridge health condition based on residuals: step 3}

Step 3 is to make a decision on bridge health condition based on residuals utilizing 95\% confidence interval and the Bayesian hypothesis testing. In the context of bridge health monitoring, we assumed that the residuals follow a normal distribution (Jiang and Mahadevan 2008). The residuals are differences between the $\mathrm{DI}_{\mathrm{ob}}$ in the step 1 and the $\mathrm{DI}_{\mathrm{pr}}$ from the Bayesian regression in the step 2. 


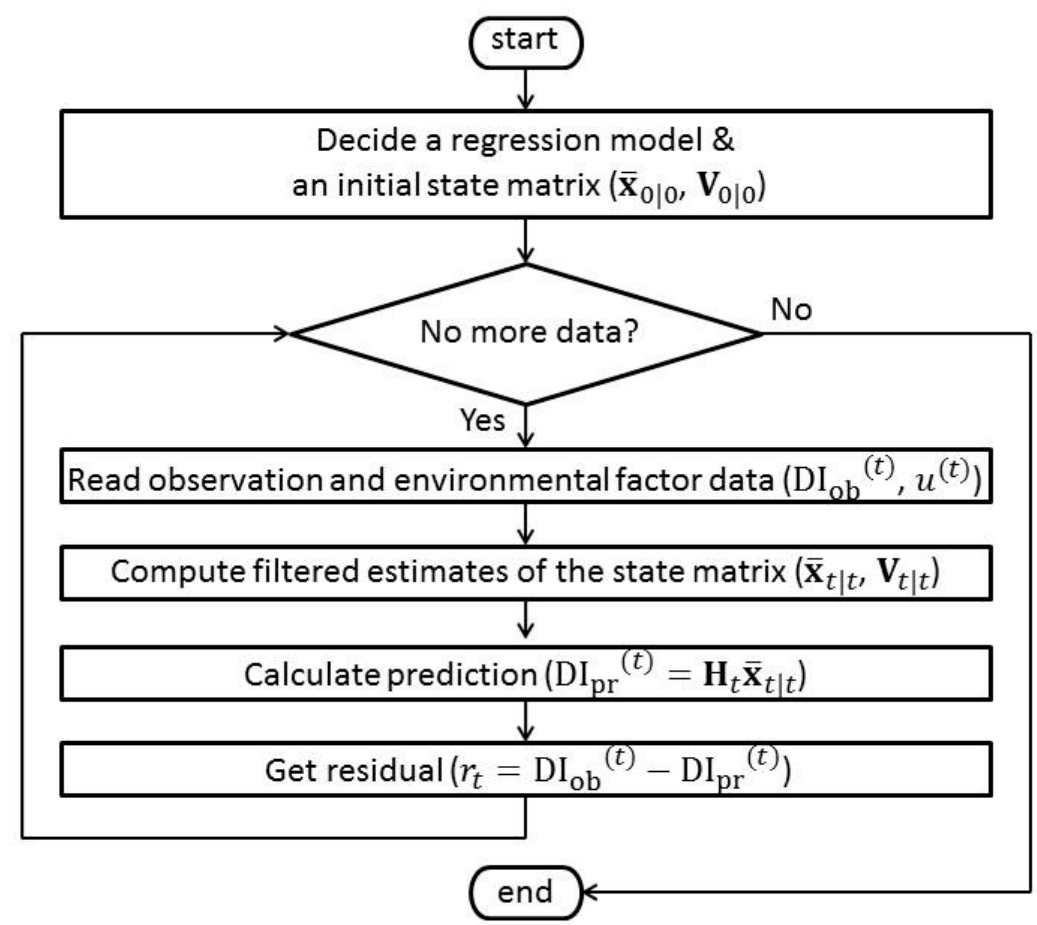

Fig. 1 Flowchart of the Bayesian regression analysis to consider environmental changes

\subsubsection{Decision-making utilizing 95\% confidence interval}

This study adopts a fitting probability of the residuals within $95 \%$ confidence interval. The threshold for the 95\% confidence interval is represented by $\mu \pm 1.96 \sigma$, where $\mu$ and $\sigma$ indicate the mean and standard deviation of the residuals respectively. The $95 \%$ confidence interval means that the interval contains $95 \%$ of the residuals of observations taken from the healthy bridge. Therefore, if the fitting probability is much less than $95 \%$, there might be some changes in the bridge health condition.

\subsubsection{Decision-making utilizing the Bayesian hypothesis testing}

In Bayesian statistics, it is possible to compute the probability of a hypothesis conditionally on observed data. Quantities that are conditional on observed data are called posterior, and the posterior odds is used for comparing hypothesis as shown in Eq. (22).

$$
\text { Post }_{\text {odds }}=\frac{p\left(\mathrm{H}_{1} \mid \mathbf{D}\right)}{p\left(\mathrm{H}_{0} \mid \mathbf{D}\right)}
$$

If the null hypothesis $\left(\mathrm{H}_{0}\right)$ is defined as 'healthy' and the alternate hypothesis $\left(\mathrm{H}_{1}\right)$ is defined as 'damage'. The posterior odds are obtained by utilizing priors and marginal likelihoods as follows.

$$
\text { Post }_{\text {odds }}=\frac{p\left(\mathrm{H}_{1} \mid \mathbf{D}\right)}{p\left(\mathrm{H}_{0} \mid \mathbf{D}\right)}=\frac{p\left(\mathbf{D} \mid \mathrm{H}_{1}\right)}{p\left(\mathbf{D} \mid \mathrm{H}_{0}\right)} \times \frac{p\left(\mathrm{H}_{1}\right)}{p\left(\mathrm{H}_{0}\right)}
$$


where $\mathbf{D}$ refers to the data obtained during monitoring, $p\left(\mathrm{H}_{1}\right) / p\left(\mathrm{H}_{0}\right)$ indicates the prior odds, and $p\left(\mathbf{D} \mid \mathrm{H}_{1}\right)$ and $p\left(\mathbf{D} \mid \mathrm{H}_{0}\right)$ are called the marginal likelihoods.

We call the ratio of the marginal likelihoods as Bayes factor $(B)$, which is defined as the ratio of likelihood of the two scenarios 'damage' and 'healthy' as follows (e.g. Kass and Raftery 1995).

$$
\begin{aligned}
B & =\frac{p\left(\mathbf{D} \mid \mathrm{H}_{1}\right)}{p\left(\mathbf{D} \mid \mathrm{H}_{0}\right)} \\
& =\frac{\int p\left(\mathbf{D} \mid \theta_{1}, \mathrm{H}_{1}\right) p\left(\theta_{1} \mid \mathrm{H}_{1}\right) \mathrm{d} \theta_{1}}{\int p\left(\mathbf{D} \mid \theta_{0}, \mathrm{H}_{0}\right) p\left(\theta_{0} \mid \mathrm{H}_{0}\right) \mathrm{d} \theta_{0}}
\end{aligned}
$$

where $\theta_{0}$ and $\theta_{1}$ are parameters under $\mathrm{H}_{0}$ and $\mathrm{H}_{1}$ respectively.

If the Bayes factor is greater than 1, it implies that the data favor the hypothesis $\mathrm{H}_{1}$ and hence suggests that there is damage. If the Bayes factor is less than 1 , then there is no damage. According to Jeffreys (1998), a Bayes factor such that $1<B<3$ is 'barely worth mentioning', $3<B<10$ is 'substantial', $10<B<30$ is 'strong', $30<B<100$ is 'very strong', and $B>100$ is 'decisive'. In other words, $B<1$ is 'nothing (no damage)', $1<B<3$ is 'very small', $3<B<10$ is 'small', $10<B<30$ is 'strong', $30<B<100$ is 'very strong' and $B>100$ is 'decisive (damage)'. In practical applications, it might provide a threshold to decide an inspection, e.g. an emergency inspection if the Bayes factor gets more than 100 continuously, although more comprehensive studies are needed.

\section{Long-term monitoring on an in-service plate-Gerber bridge}

This study utilizes data monitored at a short span steel girder bridge recorded over a one year period. The seven-span plate-Gerber bridge shown in Fig. 2 is the observed bridge, which is located on a busy national road in Japan. The bridge properties are summarized in Table 1 . The plan view with sensor locations on the observation span is shown in Fig. 3. Therein, UA-1, UA-2, DA-1 and DA-2 stand for accelerometers (high sensitivity accelerometers made by Tokyo Sokki Kenkyujo Co., Ltd.) to measure acceleration responses of steel girders on up (UA) and down (DA) lanes. The sampling rate was $200 \mathrm{~Hz}$ for acceleration measurements. Thermometers (thermocouple thermometers made by Tokyo Sokki Kenkyujo Co., Ltd.) are denoted by T-5 and T-6. Temperature is measured once every hour. A BWIM system (Moses 1979 and Heng et al. 2011) is installed in the bridge, and this study also utilizes the vehicle's whole weight estimated by the BWIM system.

Table 1 Properties of the observation bridge

\begin{tabular}{|llr|}
\hline Construction year & & 1960 \\
Bridge length (m) & 186.4 \\
Span length (m) & Hanging girder & 16.0 \\
& Anchorage girder & 40.8 \\
Width (m) & & 8.0 \\
\hline
\end{tabular}



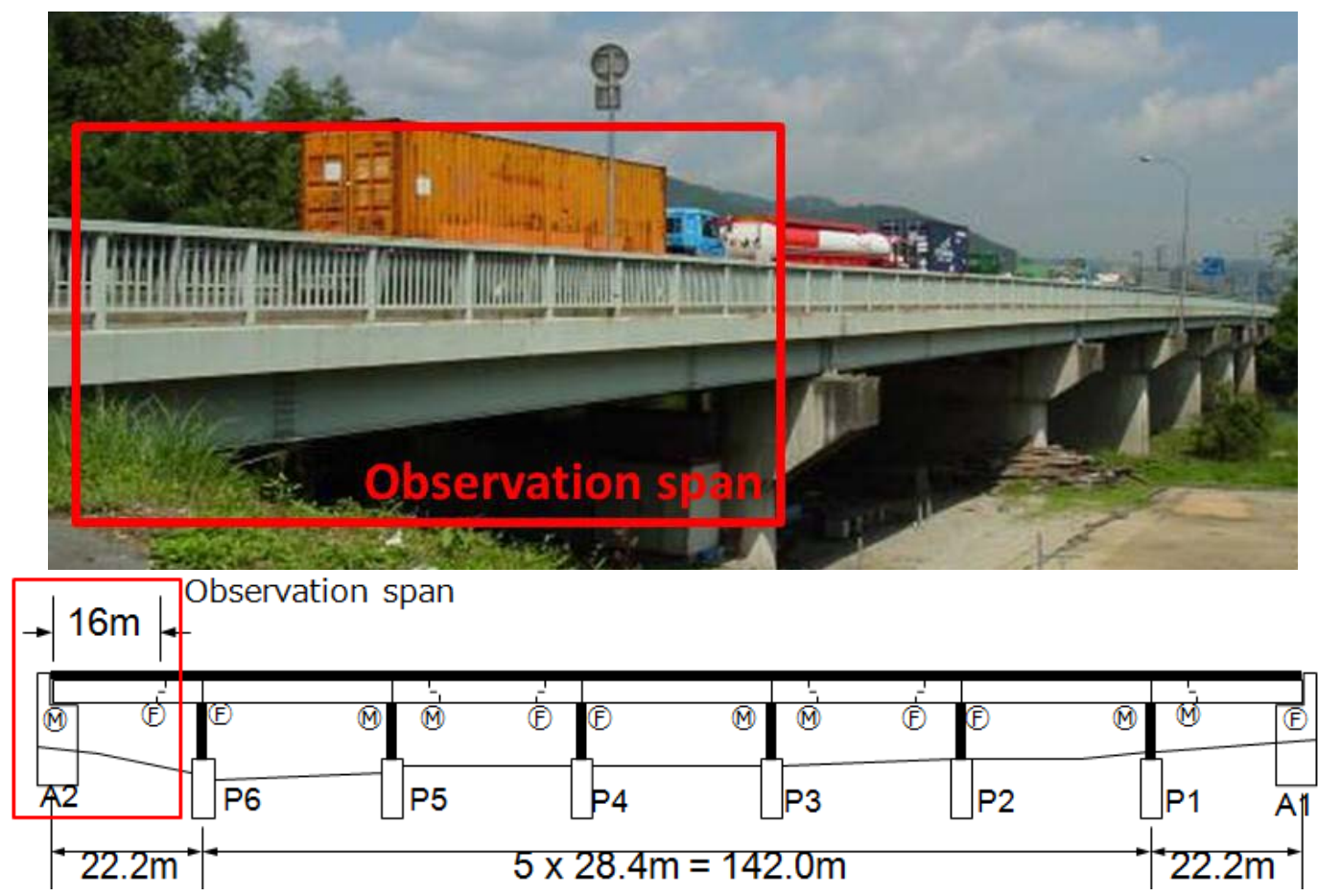

Fig. 2 Observation bridge.

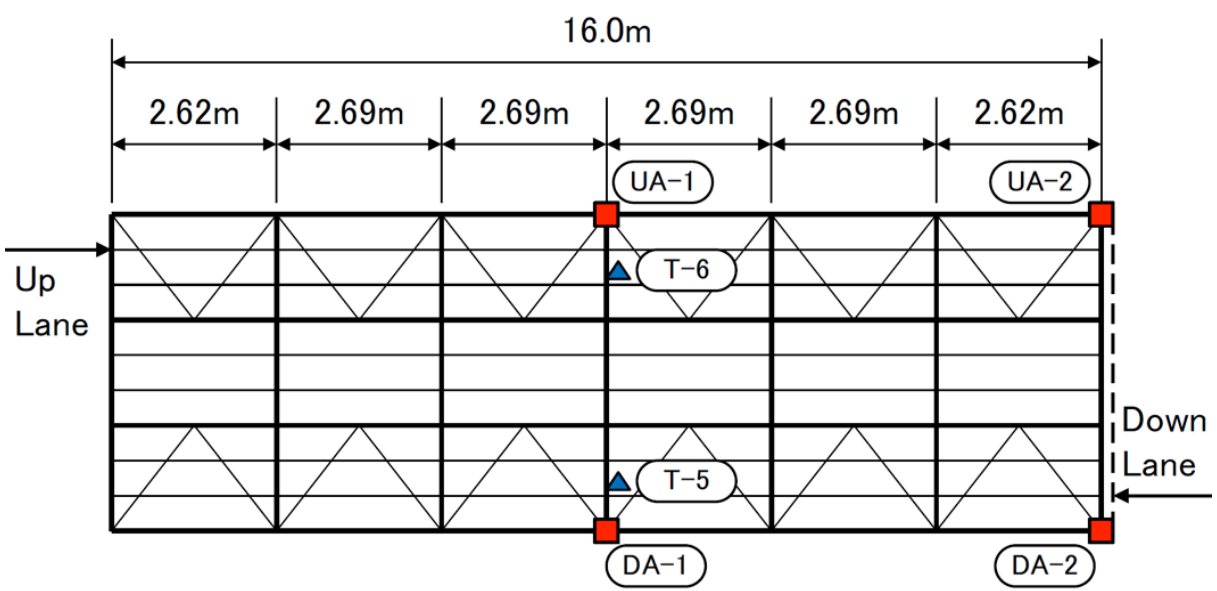

Fig. 3 Sensor locations on the observation span.

This study focuses on the effects of time-varying temperature and vehicle weight measured at 7:00, 13:00 and 19:00 on every Wednesday and Sunday for about one year ( $6^{\text {th }}$ August 2008 to $21^{\text {st }}$ June 2009); the number of measurements is 276. This study utilized the data measured at 7:00, 13:00 and 19:00 on every Wednesday and Sunday represent the data measured in the morning, afternoon and night every weekday and weekend in order to reduce time consuming data analysis in the context of a preliminary study. A noteworthy point is that all the data is taken from the healthy bridge since no damage and deterioration was reported during the monitoring period. 


\section{Application and discussion}

\subsection{Regression analysis to consider environmental changes}

Three different data combinations summarized in Table 2 are considered to investigate the influence of temperature and vehicle weight as environmental and operational factors: Case 1 is to utilize acceleration, temperature and vehicle weight data considering temperature and vehicle weight as environmental and operational factors; Case 2 is to utilize acceleration and temperature data considering temperature as environmental factor; and Case 3 is to utilize only acceleration data without considering environmental and operational effects.

Table 2 Three cases to consider in this study

\begin{tabular}{|c|c|c|}
\hline & Considered factors & Utilized data \\
\hline Case 1 & Temperature\& vehicle weight & Acceleration, temperature \& vehicle weight \\
\hline Case 2 & Temperature & Acceleration \& temperature \\
\hline Case 3 & Not considered & Acceleration \\
\hline
\end{tabular}

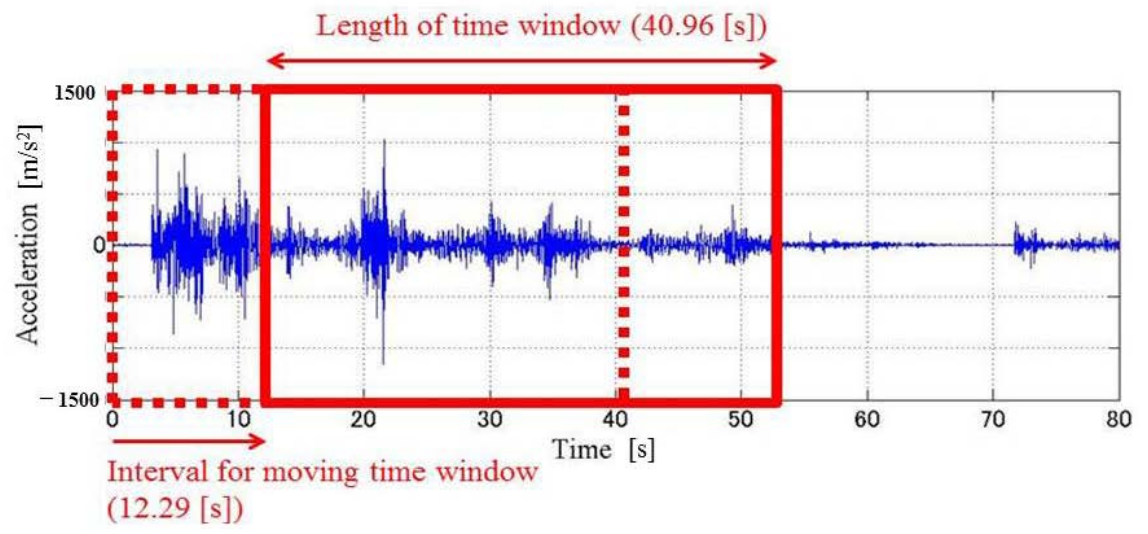

Fig. 4 Time windows to identify the $\mathrm{DI}_{\mathrm{ob}}$ 's from acceleration measured at UA-1

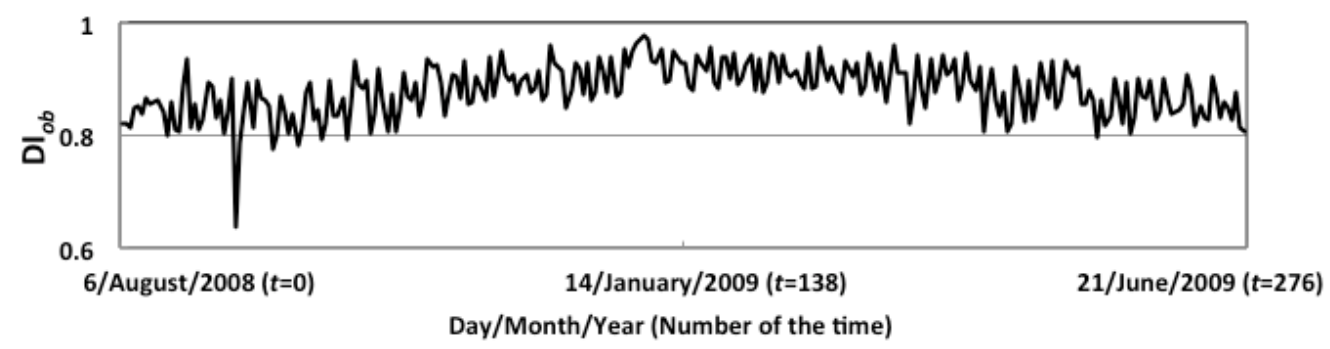

Fig. $5 \mathrm{DI}_{\mathrm{ob}}$ 's identified at the sensor of UA-1 

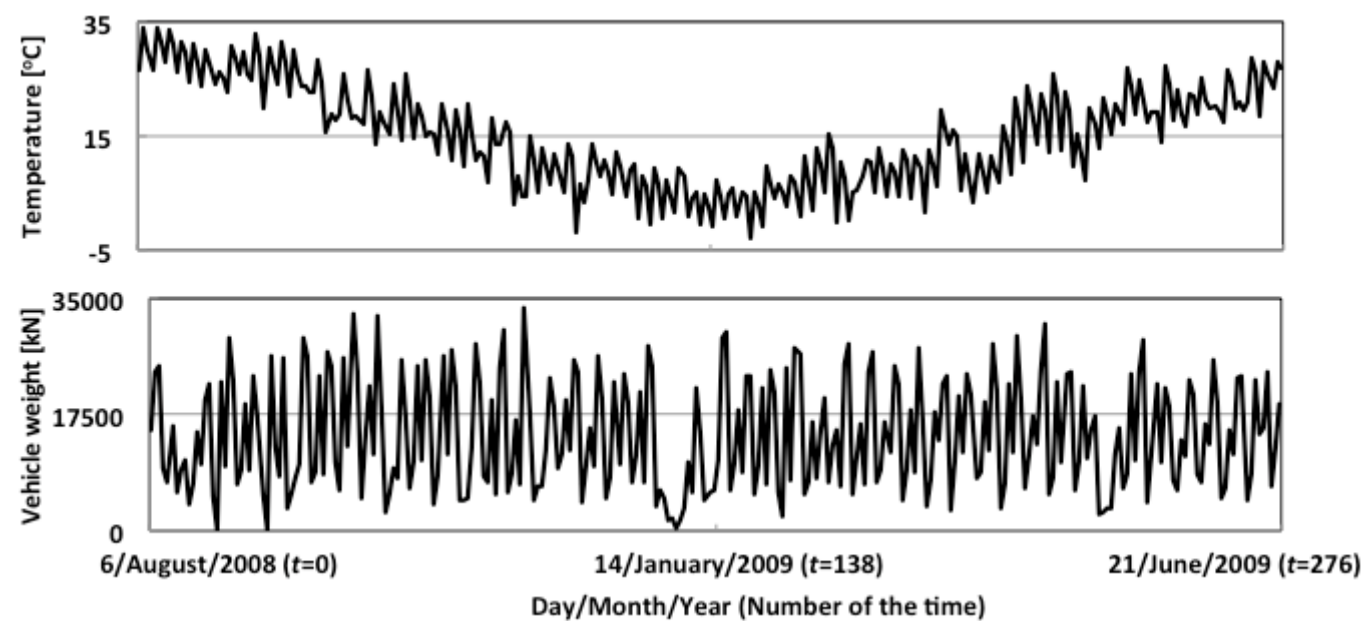

Fig. 6 Temperature measured at the sensor of T-6 and vehicle weight estimated by the BWIM system on up lane

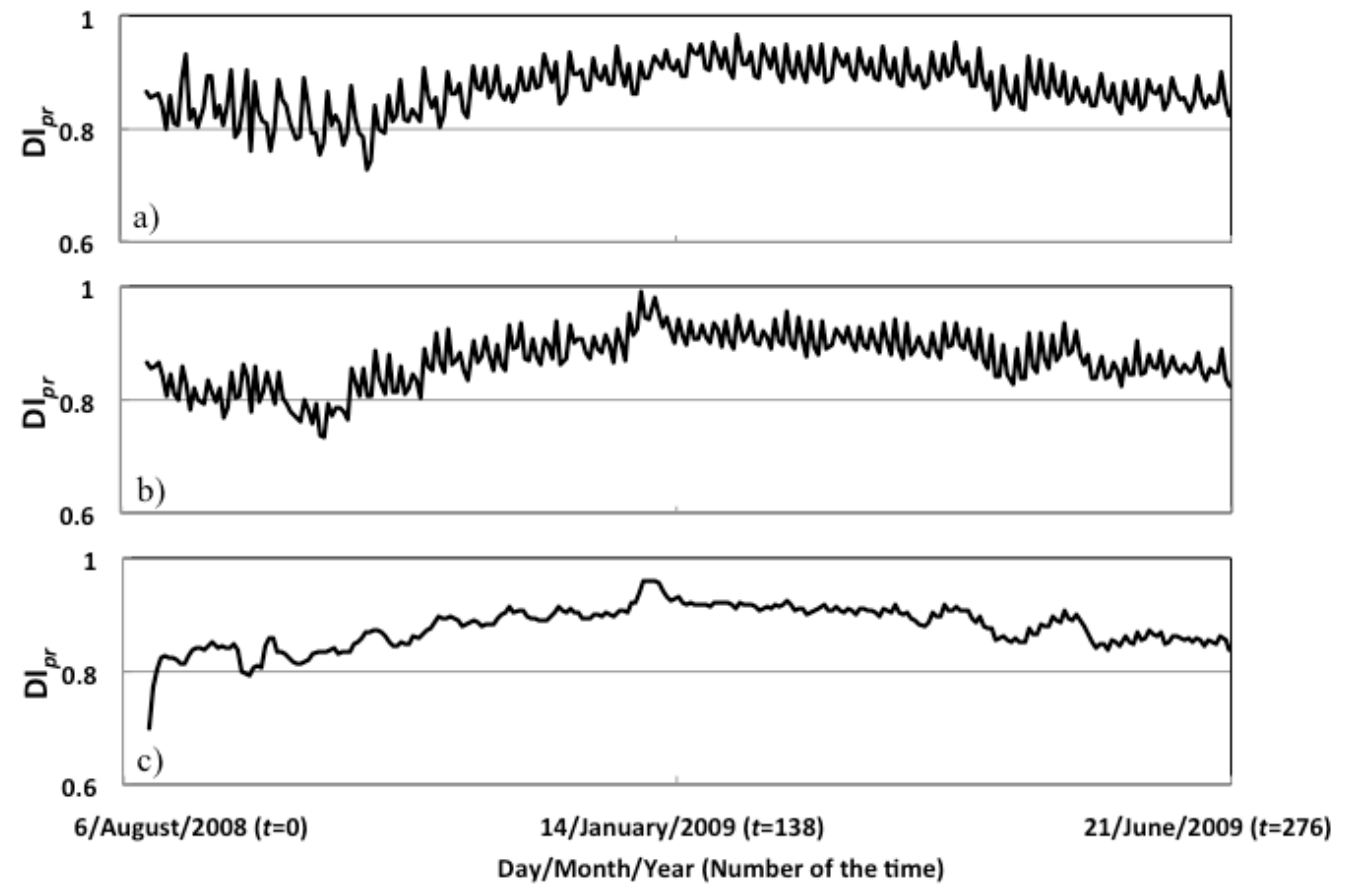

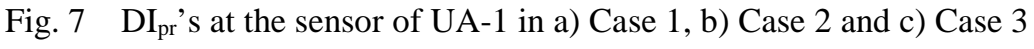

This study identifies $100 \mathrm{DI}_{\mathrm{ob}}$ 's from blocks of acceleration data obtaining by means of moving time windows as shown in Fig. 4. This study adopted 40.96s as the moving window length following a preliminary investigation considering data 20.48s, 40.96s and 81.92s which showed similar trends with each other. For the overlap window, a moving window with $70 \%$ overlap (28.67s, in other words 12.29s for un-overlap window length) was adopted in this study. 


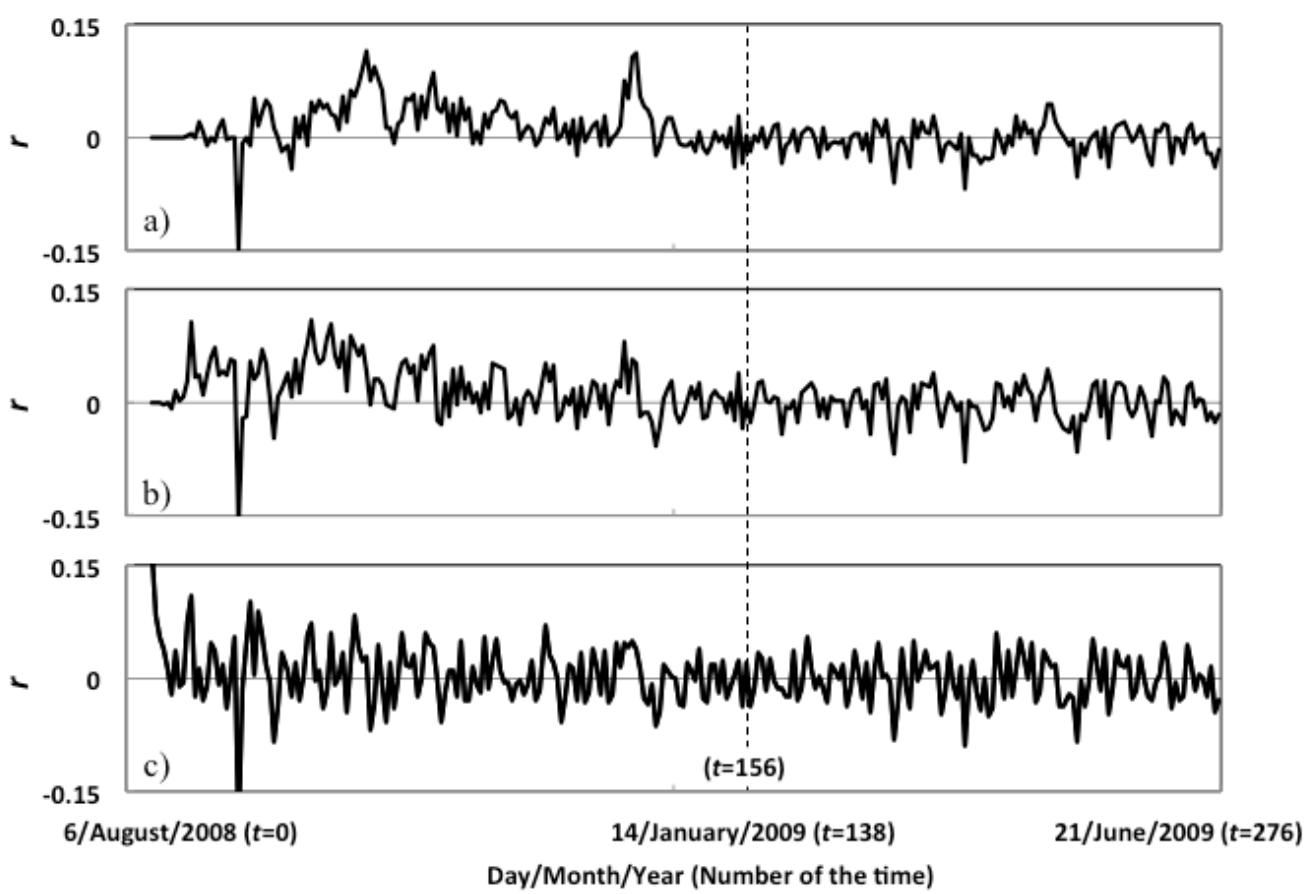

Fig. 8 Residuals at the sensor of UA-1 in a) Case 1, b) Case 2 and c) Case 3

The $\mathrm{DI}_{\mathrm{ob}}$ 's identified at the sensors of UA-1 are shown in Fig. 5. The temperature measured at the sensor of T-6 and vehicle weight estimated by the BWIM system on the up lane are shown in Fig. 6. This study considered sums of weights of vehicles traveling on the bridge during the time block that is used in estimating the $\mathrm{DI}_{\mathrm{pr}}$. Changes in the $\mathrm{DI}_{\mathrm{ob}}$ 's and temperatures during about one year were about 0.3 and 40 degrees Celsius respectively. Those changes are about $34 \%$ of mean $\mathrm{DI}_{\mathrm{ob}}(0.88)$ and about $38 \%$ of mean temperature (15.18 degrees Celsius). It is also apparent that the lower the temperatures is, the greater the $\mathrm{DI}_{\mathrm{ob}}$ 's become.

The potential reason for the $\mathrm{DI}_{\mathrm{ob}}$ increase with lower temperature is that the $\mathrm{DI}_{\mathrm{ob}}$ has similar feature with frequencies that increase with lower temperature. The changes in the $\mathrm{DI}_{\mathrm{ob}}$ 's (even frequencies) depending on temperatures might be caused by changes in the boundary condition at the supports of the bridge caused due to varying temperatures and roller supports that do not function well.

Fig. 7 shows $\mathrm{DI}_{\mathrm{pr}}$ 's at the sensor of UA-1 in Cases 1,2 and 3. Fig. 8 shows the residuals at the sensor of UA-1 in Cases 1, 2 and 3. The residuals converge within bias of \pm 0.05 after about 156 times $(t=156)$ elapsed from the start.

Table 3 shows the fitting probability of the residuals after $t=156$ in Cases 1,2 and 3 at the sensors of UA-1, UA-2, DA-1 and DA-2. This study adopts the $95 \%$ confidence interval of the residuals after $t=156$ in Case 1 as threshold of the fitting probability. It is found that the fitting probability in Cases 1 and 2 are greater than that in Case 3 at the sensors of UA-1, UA-2 and DA-2. It demonstrated that the regression analysis considering environmental and operational changes (Case 1) leads to more accurate results than that without considering environmental and 
operational changes (Case 3). It is also obvious that the fitting probability in Case 1 is greater than that in Case 2 at the sensors of UA-1. It demonstrated that the regression analysis considering both temperature and vehicle weight as environmental and operational factors (Case 1) leads to more accurate results than that considering only temperature as an environmental factor (Case 2), even though the differences are negligible except UA-1 in which UA-1 is located on the entering span of the up lane and easily affected by large dynamic wheel loads of the vehicles activated when the vehicles pass on the expansion joint of the entering span (Kim et al. 2007). From this result, it would be reasonable to consider only temperature under condition less affected by passing vehicles.

Table 3 Fitting probability of residuals after $t=156$ in Cases 1,2 and 3 within $95 \%$ confidence interval regarding Case 1

\begin{tabular}{|c|cccc|}
\hline & UA-1 & UA-2 & DA-1 & DA-2 \\
\hline Case 1 & $95.9 \%$ & $92.6 \%$ & $92.6 \%$ & $93.4 \%$ \\
\hline Case 2 & $92.6 \%$ & $92.6 \%$ & $92.6 \%$ & $93.4 \%$ \\
\hline Case 3 & $79.3 \%$ & $89.3 \%$ & $93.4 \%$ & $90.9 \%$ \\
\hline
\end{tabular}

\subsection{Bayes factor for decision-making on anomaly from residuals}

As an example of applying the Bayesian hypothesis testing, this study assumes that the null hypothesis is defined by Eq. (25) and the alternate hypothesis is defined by Eq. (26) (Sankararaman and Mahadevan 2011).

$$
\begin{aligned}
& \mathrm{H}_{0}: \mu=0 \\
& \mathrm{H}_{1}: \mu \neq 0
\end{aligned}
$$

where $\mu$ denotes the mean value of the residual shown in Eq. (12). (27).

This study adopts the equation for $B$ proposed by Jiang and Mahadevan (2008) shown in Eq.

$$
\begin{aligned}
B & =\frac{\int p\left(\mathbf{D} \mid \mu, \mathrm{H}_{1}\right) p\left(\mu \mid \mathrm{H}_{1}\right) \mathrm{d} \mu}{p(\mathbf{D})} \\
& =\frac{\left(\frac{1}{2 \pi \sigma^{2}}\right)^{\frac{N}{2}} \frac{1}{\sqrt{N+1}} \exp \left(-\frac{1}{2 \sigma^{2}} \sum_{i=1}^{N}\left(r_{i}-\mu\right)^{2}\right) \exp \left(-\frac{N \mu^{2}}{2(N+1) \sigma^{2}}\right)}{\left(\frac{1}{2 \pi \sigma^{2}}\right)^{\frac{N}{2}} \exp \left(-\frac{1}{2 \sigma^{2}} \sum_{i=1}^{N} r_{i}^{2}\right)} \\
& =\frac{1}{\sqrt{N+1}} \exp \left(\frac{N^{2} \mu^{2}}{2(N+1) \sigma^{2}}\right)
\end{aligned}
$$

where $N$ denotes the number of the data and $r_{i}$ represents the $i$-th residual. 
Table 4 shows Bayes factor $(B)$ of the residuals after $t=156$ for Cases 1,2 and 3. The Bayes factors were $0<B<1$ except UA-1 of Case 1 . Therefore, the probability of damage of the observation bridge is 'no damage', which is natural since data from healthy bridge were considered. At UA-1 of Case 1, greater mean values with less standard deviation of the residuals than other cases would result in relatively bigger Bayes factor (see Eq. (27)).

Table 5 shows the means and standard deviations of the residuals after $t=156$ in Cases 1, 2 and 3. Observations from Table 4 and Table 5 show that the greater the mean values with less standard deviation of the residuals led to bigger Bayes factors.

Table 4 Bayes factor of residuals after $t=156$ in Bayesian hypothesis testing in Cases 1,2 and 3

\begin{tabular}{|c|cccc|}
\hline & UA-1 & UA-2 & DA-1 & DA-2 \\
\hline Case 1 & 1.26 (very small) & 0.10 (no damage) & 0.11 (no damage) & 0.13 (no damage) \\
\hline Case 2 & 0.17 (no damage) & 0.09 (no damage) & 0.14 (no damage) & 0.16 (no damage) \\
\hline Case 3 & 0.13 (no damage) & 0.16 (no damage) & 0.11 (no damage) & 0.10 (no damage) \\
\hline
\end{tabular}

Table 5 Mean values and standard deviations of the residuals after $t=156$ in Cases 1,2 and 3

\begin{tabular}{|l|cc|cc|cc|cc|}
\hline & \multicolumn{2}{|c|}{ UA-1 } & \multicolumn{2}{c|}{ UA-2 } & \multicolumn{2}{c|}{ DA-1 } & \multicolumn{2}{c|}{ DA-2 } \\
\cline { 2 - 9 } & $\mu$ & $\sigma$ & $\mu$ & $\sigma$ & $\mu$ & $\sigma$ & $\mu$ & $\sigma$ \\
\hline Case 1 & -0.0042 & 0.020 & -0.0013 & 0.038 & 0.0012 & 0.020 & 0.0027 & 0.034 \\
\hline Case 2 & -0.0025 & 0.024 & -0.0010 & 0.042 & 0.0017 & 0.021 & 0.0035 & 0.035 \\
\hline Case 3 & -0.0025 & 0.030 & -0.0045 & 0.047 & 0.0012 & 0.021 & 0.0014 & 0.038 \\
\hline
\end{tabular}

\section{Conclusions}

This study investigated a way to consider changes in temperature and vehicle weight as environmental and operational factors for long-term vibration-based BHM by applying a Bayesian approach to long-term monitoring data on an in-service plate-Gerber bridge. The Bayesian approach consists of three steps: step 1 is to identify damage-sensitive features from coefficients of the AR model utilizing bridge accelerations; step 2 is to make a regression analysis of the damagesensitive features to consider environmental and operational changes by means of the Bayesian regression; step 3 is to make a decision based on the residuals utilizing 95\% confidence interval and the Bayesian hypothesis testing. Observations through this study could be summarized as follows.

(1) The Bayesian regression considering environmental and operational changes led to more accurate results than that without considering environmental and operational changes.

(2) Regression considering only temperature change was comparable with the regression considering changes in temperature and vehicles on the bridge. From this result, it could be concluded that considering only temperature as an environmental factor would be useful in vibration-based long-term monitoring.

(3) In the Bayesian hypothesis testing utilizing data from the healthy bridge, the damage probability of the bridge was judged as no damage. However greater mean values with less standard deviation of residuals led to a bigger Bayes factor, which suggests further 
investigations on establishing a proper hypothesis, for instance " $\mathrm{H}_{0}: \mu=0 \pm \Delta$ " where $\Delta$ denotes a bias of mean residuals, etc.

\section{Acknowledgments}

This study is partly sponsored by JSPS, Grant-in-Aid for Scientific Research (B) under project No. 24360178. Such financial aids are gratefully acknowledged.

\section{References}

Akaike, H. (1974), “A new look at the statistical model identification”, IEEE Transactions on Automatic Control, 19(6), 716-723.

Deraemaeker, A., Reynders, E., De Roeck, G. and Kullaa, J. (2007), "Vibration-based structural health monitoring using output-only measurements under changing environment”, Mech. Systems Signal Processing, 22(1), 34-56.

Dilena, M. and Morassi, A. (2011), “Dynamic testing of damaged bridge”, Mech. Systems Signal Processing, 25, 1485-1507.

Doebling, S.W., Farrar, C.R., Prime, M.B. and Shevitz, D.W. (1996), "Damage identification and health monitoring of structural and mechanical systems from changes in their vibration characteristics: A literature review", Los Alamos National Laboratory Report, LA-3070-MS.

Cunha, A., Caetano, E., Magalhaes, F. and Moutinho, C. (2013), "Recent perspectives in dynamic testing and monitoring of bridges”, Struct. Control Health Monitoring, 20(6), 853-877.

Heng, S., Oshima, Y. and Kawano, H. (2011), "One Year Monitoring of Bridge Frequency and Traffic Load on a Road Bridge”, Proceedings of 24th KKCNN Symposium on Civil Engineering.

Jeffreys, H. (1998), Theory of Probability, Oxford University Press Inc., New York, First published in the Oxford Classics series.

Jiang, X. and Mahadevan, S. (2008), "Bayesian wavelet methodology for structural damage detection”, Struct. Control Health Monitoring, 15, 974-991.

Kass, R. and Raftery, A. (1995), "Bayes factors”, J. American Statistical Association, 90(430):773-795.

Kim, C.W., Kawatani, M. and Kwon, Y.R. (2007), "Impact coefficient of reinforced concrete slabs on a steel girder bridge”, Eng. Struct., 29(4), 576-590.

Kim, C.W., Sakakibara, T., Isemoto, R., Salpisoth, H., Oshima, Y. and Sugiura, K. (2011), "One year vibration monitoring of a short span bridge under in-service environments", Proceedings of 5th International Conference on Structural Health Monitoring of Intelligent Infrastructure (SHMII-5), Cancun, Mexico (CD-ROM).

Kim, C.W., Kawatani, M. and Hao, J. (2012), "Modal parameter identification of short span bridges under a moving vehicle by means of multivariate AR model”, Struct. Infrastructure Eng., 8(5), 459-472.

Kim, C.W., Kitauchi, S., Sugiura, K. and Kawatani, M. (2013a), “A year-long monitoring using in-service vibration data from a multi-span plate-Gerber bridge”, Proceedings of Life-Cycle and Sustainability of Civil Infrastructure Systems, Strauss, Frangopol and Bergmeister (Eds.), 252-259.

Kim, C.W., Isemoto, R., Sugiura, K. and Kawatani, M. (2013b), "Structural fault detection of bridges based on linear system parameter and MTS method”, J. JSCE, 1(1), 32-43.

Kim, C.W., Isemoto, R., Sugiura, K. and Kawatani, M. (2013c), "Linear system parameter as an indicator for structural diagnosis of short span bridges”, Smart Struct. Systems, An Int J., 11(1), 1-17.

Kitagawa, G. and Gersch, W. (1984), "A smoothness priors-state space modeling of time series with trend and seasonality”, J. American Statistical Association, 79, 378-389. 
Moses, F. (1979), “Weigh-In-Motion System Using Instrumented Bridges”, Transportation Eng. J., 105, TE3.

Nair, K.K., Kiremidjian, A.S. and Law, K.H. (2006), “Time series-based damage detection and localization algorithm with application to the ASCE benchmark structure”, Sound Vibration, 291, 349-368.

Peeters, B. and De Roeck, G. (2001), “One-year monitoring of the Z24-Bridge: environmental effects versus damage events”, Earthquake Eng. Struct. Dynamics, 30, 149-171.

Sankararaman, S. and Mahadevan, S. (2011), "Bayesian methodology for diagnosis uncertainty quantification and health monitoring”, Struct. Control Health Monitoring, 19, 88-106.

Sohn, H., Worden, K. and Farrar, C.R. (2003), "Statistical damage classification under changing environmental and operational conditions”, Intelligent Material Systems Struct., 13, 153-160. 